TRANSACTIONS OF THE

AMERICAN MATHEMATICAL SOCIETY

Volume 350, Number 11, November 1998, Pages 4569-4591

S 0002-9947(98)02162-X

\title{
THE SANTALÓ-REGIONS OF A CONVEX BODY
}

\author{
MATHIEU MEYER AND ELISABETH WERNER
}

\begin{abstract}
Motivated by the Blaschke-Santaló inequality, we define for a convex body $K$ in $\mathbf{R}^{n}$ and for $t \in \mathbf{R}$ the Santaló-regions $S(K, t)$ of $K$. We investigate the properties of these sets and relate them to a concept of affine differential geometry, the affine surface area of $K$.
\end{abstract}

Let $K$ be a convex body in $\mathbf{R}^{n}$. For $x \in \operatorname{int}(K)$, the interior of $K$, let $K^{x}$ be the polar body of $K$ with respect to $x$. It is well known that there exists a unique $x_{0} \in \operatorname{int}(K)$ such that the product of the volumes $|K|\left|K^{x_{0}}\right|$ is minimal (see for instance [Sch]). This unique $x_{0}$ is called the Santaló-point of $K$.

Moreover, the Blaschke-Santaló inequality says that $|K|\left|K^{x_{0}}\right| \leq v_{n}^{2}$ (where $v_{n}$ denotes the volume of the $n$-dimensional Euclidean unit ball $B(0,1)$ ) with equality if and only if $K$ is an ellipsoid.

For $t \in \mathbf{R}$ we consider here the sets

$$
S(K, t)=\left\{x \in K: \frac{|K|\left|K^{x}\right|}{v_{n}^{2}} \leq t\right\} .
$$

Following E. Lutwak, we call $S(K, t)$ a Santaló-region of $K$.

Observe that it follows from the Blaschke-Santaló inequality that the Santalópoint $x_{0} \in S(K, 1)$, and that $S(K, 1)=\left\{x_{0}\right\}$ if and only if $K$ is an ellipsoid. Thus $S(K, t)$ has non-empty interior for some $t<1$ if and only if $K$ is not an ellipsoid.

In the first part of this paper we show some properties of $S(K, t)$ and give estimates on the "size" of $S(K, t)$. This question was asked by E. Lutwak.

In the second part we show how $S(K, t)$ is related to the affine surface area of $K$.

The affine surface area $a s(K)$ is originally a notion of differential geometry. For a convex body $K$ in $\mathbf{R}^{n}$ with sufficiently smooth boundary $\partial K$ it is defined as

$$
a s(K)=\int_{\partial K} \kappa(x)^{\frac{1}{n+1}} d \mu(x),
$$

where $\kappa(x)$ is the Gaussian curvature at $x \in \partial K$ and $\mu$ is the surface measure on $\partial K$. The affine surface area is invariant under affine transformations with determinant 1. It arises naturally in questions concerning the approximation of convex bodies by polytopes (see $[\mathrm{G}]$ ) and in a priori estimates of PDE's ([Lu-O]).

Received by the editors October 25, 1996.

1991 Mathematics Subject Classification. Primary 52A20; Secondary 52A38.

Key words and phrases. Blaschke-Santaló inequality, affine surface area.

Supported by a grant from the National Science Foundation.

The paper was written while both authors were at MSRI.

(C)1998 American Mathematical Society 
It has been one of the aims of convexity theory to extend the notions of differential geometry and (for instance) of affine surface area to arbitrary convex bodies without any smoothness assumptions on the boundary.

Within the last few years four different extensions have been given (due to Leichtweiss [L1], Lutwak [Lu], Schütt-Werner [S-W] and Werner [W]), and it was shown that they all coincide ([S1], [D-H]).

We give here another such extension, arising again from a completely different context. It will also follow that this new extension coincides with the others.

The authors wish to thank MSRI for its hospitality, and the organizers of the special semester in Convex Geometry and Geometric Functional Analysis at MSRI for inviting them. It was during our stay there that the paper was written.

Unless stated otherwise we will always assume that a convex body $K$ in $\mathbf{R}^{n}$ has its Santaló-point at the origin. Then 0 is the center of mass of the polar body $K^{0}$, which may be written as

$$
\int_{K^{0}}\langle x, y\rangle d y=0 \quad \text { for every } \quad x \in \mathbf{R}^{n} .
$$

By $|K|$ we denote the $n$-dimensional volume of $K . h_{K}$ is the support function of $K$. If $K$ is centrally symmetric, $\|.\|_{K}$ is the norm on $\mathbf{R}^{n}$ that has $K$ as its unit ball. By $\|$.$\| we denote the standard Euclidean norm on \mathbf{R}^{n}$, and $\langle\cdot$,$\rangle is the usual inner$ product on $\mathbf{R}^{n}$. $B(a, r)$ is the $n$-dimensional Euclidean ball with radius $r$ centered at $a$. For $x \in K, K^{x}=(K-x)^{0}=\left\{y \in \mathbf{R}^{n}:\langle y, z-x\rangle \leq 1\right.$ for all $\left.z \in K\right\}$ is the polar body of $K$ with respect to $x$; $K^{0}$ denotes the polar body with respect to the Santaló-point. Moreover for $u \in S^{n-1}$ we will denote by $\phi_{K}^{u}(y)$ or for short by $\phi(y)$ the $(n-1)$-dimensional volume of the sections of $K$ orthogonal to $u$; that is,

$$
\phi(y)=\phi_{K}^{u}(y)=|\{z \in K:\langle z, u\rangle=y\}| .
$$

\section{Properties of the Santaló-Regions}

We start by listing some of the properties of $S(K, t)$. Recall that for $\delta>0, \delta$ small enough, $K_{\delta}$ is said to be a (convex) floating body of $K$, if it is the intersection of all halfspaces whose defining hyperplanes cut off a set of volume $\delta$ of $K$ ([S-W]). More precisely, for $u \in S^{n-1}$ and for $0<\delta$ let $a_{\delta}^{u}$ be defined by

$$
\left|\left\{x \in K:\langle x, u\rangle \geq a_{\delta}^{u}\right\}\right|=\delta .
$$

Then $K_{\delta}=\bigcap_{u \in S^{n-1}}\left\{x \in K:\langle x, u\rangle \leq a_{\delta}^{u}\right\}$.

In the following proposition we consider only those $t \in \mathbf{R}$ for which $S(K, t) \neq \emptyset$.

Proposition 1. Let $K$ be a convex body in $\mathbf{R}^{n}$. Then:

(i) $S(K, t)$ is strictly convex for all $t$.

(ii) $S(A(K), t)=A(S(K, t))$ for all regular affine transformations $A$, for all $t$.

(iii) The boundary of $S(K, t)$ is $C^{\infty}$ for all $t$.

(iv) $t \longmapsto S(K, t)$ is increasing and concave; that is, for all $t, s$ and for all $\alpha \in \mathbf{R}$, $0 \leq \alpha \leq 1$,

$$
S(K, \alpha t+(1-\alpha) s) \supset \alpha S(K, t)+(1-\alpha) S(K, s) .
$$


(v) For all $0<\delta<\frac{1}{2}, K_{\delta|K|}$ is contained in $S\left(K, \frac{1}{4 \delta(1-\delta)}\right)$.

Proof. We frequently use the following well known formula. For all $x \in \operatorname{int}(K)$

$$
\left|K^{x}\right|=\frac{1}{n} \int_{S^{n-1}} \frac{d \sigma(u)}{\left(h_{K}(u)-\langle u, x\rangle\right)^{n}},
$$

where $\sigma$ is the spherical Lebesgue measure.

Indeed, $\left|K^{x}\right|=\frac{1}{n} \int_{S^{n-1}} \frac{d \sigma(u)}{\left(\hat{h}_{K}(u)\right)^{n}}$, where $\tilde{h}_{K}$ is the support function of $K$ centered at $x$. Now observe that $\tilde{h}_{K}(u)=h_{K}(u)-\langle u, x\rangle$; thus (1) follows.

(i) Observe that for all $u \in S^{n-1}$ the function

$$
x \longmapsto \frac{1}{\left(h_{K}(u)-\langle x, u\rangle\right)^{n}}
$$

is convex on $\operatorname{int}(K)$. (1) then implies that

$$
x \longmapsto\left|K^{x}\right|=\frac{1}{n} \int_{S^{n-1}} \frac{d \sigma(u)}{\left(h_{K}(u)-\langle u, x\rangle\right)^{n}}
$$

is convex. In fact the function

$$
x \longmapsto\left|K^{x}\right|=\frac{1}{n} \int_{S^{n-1}} \frac{d \sigma(u)}{\left(h_{K}(u)-\langle u, x\rangle\right)^{n}}
$$

is strictly convex, as for $x, y \in \operatorname{int}(K), x \neq y$,

$$
\sigma\left(\left\{u \in S^{n-1}:\langle u, x\rangle=\langle u, y\rangle\right\}\right)=0 .
$$

Therefore (i) follows.

(ii) Let $A$ be a one-to-one affine transformation. We can write $A=L+a$, where $L$ is a one-to-one linear transformation and $a$ is a vector in $\mathbf{R}^{n}$. Then

Hence

$$
\begin{gathered}
(A(K))^{y}=\left\{x \in \mathbf{R}^{n}:\langle x, A z-y\rangle \leq 1 \quad \text { for all } \mathrm{z} \in K\right\} \\
=\left\{x \in \mathbf{R}^{n}:\left\langle L^{*} x, z-A^{-1} y\right\rangle \leq 1 \quad \text { for all } \mathrm{z} \in K\right\} \\
=\left(L^{*}\right)^{-1}\left(K^{A^{-1}(y)}\right) .
\end{gathered}
$$

$$
\begin{gathered}
S(A K, t)=\left\{y \in A K: \frac{|A K|\left|A K^{y}\right|}{v_{n}^{2}} \leq t\right\} \\
=\left\{y \in A K: \frac{|K|\left|K^{A^{-1}(y)}\right|}{v_{n}^{2}} \leq t\right\} \\
=A(S(K, t)) .
\end{gathered}
$$

(iii) Let $K, n$ and $t$ be fixed. By (1), $\partial S(K, t)=\left\{x \in K: F(x)=\frac{n t v_{n}^{2}}{|K|}\right\}$, where $F(x)=\int_{S^{n-1}} \frac{d \sigma(u)}{\left(h_{K}(u)-\langle u, x\rangle\right)^{n}} . \quad F$ is continuous on $\operatorname{int}(K)$ with continuous partial derivatives of all orders, has a unique minimum at the Santaló-point $x_{0}$, and is convex (see (i)). Therefore (iii) follows from the implicit function theorem.

(iv) is obvious from the proof of (i).

(v) Let $\delta \in\left(0, \frac{1}{2}\right)$, and let $H$ be a hyperplane that has non-empty intersection with $K$ and is such that

$$
\left|K \cap H^{+}\right|=\delta|K|,
$$

where $H^{+}$is one of the two halfspaces determined by $H$. By definition the convex floating body $K_{\delta|K|}$ is the intersection of all the halfspaces $H^{-}$determined by all such hyperplanes $H$. 
On the other hand, by [Me-P] there exists $x \in H \cap \operatorname{int}(K)$ such that

$$
\frac{|K|\left|K^{x}\right|}{v_{n}^{2}} \leq \frac{1}{4 \delta(1-\delta)} .
$$

This means that $x \in S\left(K, \frac{1}{4 \delta(1-\delta)}\right)$. Consequently

$$
K_{\delta|K|} \subseteq S\left(K, \frac{1}{4 \delta(1-\delta)}\right) .
$$

Remark 2. (i) Proposition 1,(iv) says that $K_{\delta|K|} \subseteq S\left(K, \frac{1}{4 \delta(1-\delta}\right)$. We will show (see Proposition 14) that in the case of the convex body with sufficiently smooth boundary and positive Gaussian curvature everywhere a converse inclusion holds for $\delta$ "small".

(ii) Note also that for $K=B(0,1)$,

$$
(B(0,1))_{\delta v_{n}} \sim S\left(B(0,1), \frac{v_{n-1}}{\delta(n+1) v_{n}}\right),
$$

for $\delta$ sufficiently small. More precisely, for $\delta \leq \frac{2^{n+1} v_{n-1}}{\sqrt{e}(n+1) v_{n} n^{\frac{n+1}{2}}}$

$$
S\left(B(0,1), \frac{v_{n-1}}{\sqrt{e} \delta(n+1) v_{n}}\right) \subseteq(B(0,1))_{\delta v_{n}} \subseteq S\left(B(0,1), \frac{(\sqrt{e})^{\frac{n+1}{n-1}} v_{n-1}}{\delta(n+1) v_{n}}\right) .
$$

This follows from the forthcoming Corollary 5 and from the fact that the volume of a cap of the Euclidean unit ball of height $\Delta$ can be estimated from above by

$$
\frac{v_{n-1}}{n+1}(2 \Delta)^{\frac{n+1}{2}}
$$

and from below by

$$
\frac{v_{n-1}}{n+1}(2 \Delta)^{\frac{n+1}{2}}\left(1-\frac{\Delta}{2}\right)^{\frac{n-1}{2}}
$$

For $\delta$ "close" to $\frac{1}{2}$,

$$
(B(0,1))_{\delta v_{n}} \sim S\left(B(0,1), \frac{1}{4 \delta(1-\delta)}\right) .
$$

More precisely, let $\varepsilon \leq \frac{1}{\sqrt{n}}$ and $\frac{1}{2}>\delta \geq \frac{1}{2}-\varepsilon \frac{v_{n-1}}{\sqrt{e} v_{n}}$. Then

$$
(B(0,1))_{\delta v_{n}} \subseteq S\left(B(0,1), \frac{1}{4 \delta(1-\delta)}\right) \subseteq \frac{8 v_{n-1}}{\sqrt{n+1} v_{n}}(B(0,1))_{\delta v_{n}} .
$$

The following lemmas will enable us to compute $\left|(B(0,1))^{x}\right|$. They are also needed for Part II.

Lemma 3. Let $x \in \operatorname{int}(K)$. Then

$$
\left|K^{x}\right|=\int_{K^{0}} \frac{d y}{(1-\langle x, y\rangle)^{n+1}} .
$$

Proof. By (1) 


$$
\begin{gathered}
\left|K^{x}\right|=\frac{1}{n} \int_{S^{n-1}} \frac{d \sigma(u)}{\left(h_{K}(u)\right)^{n}\left(1-\left\langle\frac{u}{h_{K}(u)}, x\right\rangle\right)^{n}} \\
=\int_{S^{n-1}} \int_{0}^{\frac{1}{h_{K}(u)}} \frac{r^{n-1}}{(1-\langle x, r u\rangle)^{n+1}} d r d \sigma(u)=\int_{K^{0}} \frac{d y}{(1-\langle x, y\rangle)^{n+1}} .
\end{gathered}
$$

Remark. We will use Lemma 3 mostly in the following form:

Let $u \in S^{n-1}$ and $\lambda \in \mathbf{R}$ be such that $x=\lambda u \in \operatorname{int}(K)$. Then Lemma 3 says that

$$
\left|K^{x}\right|=\int_{-h_{K^{0}}(-u)}^{h_{K^{0}}(u)} \frac{\phi_{K^{0}}^{u}(t) d t}{(1-\lambda t)^{n+1}},
$$

where $\phi_{K^{0}}^{u}(t)=\left|\left\{z \in K^{0}:\langle z, u\rangle=t\right\}\right|$.

Lemma 4. (i) Let $0 \leq \alpha<1$. Then

$$
\int_{-1}^{1} \frac{\left(1-x^{2}\right)^{\frac{n-1}{2}} d x}{(1-\alpha x)^{n+1}}=\frac{2^{n}\left(\Gamma\left(\frac{n+1}{2}\right)\right)^{2}}{\left(1-\alpha^{2}\right)^{\frac{n+1}{2}} n !}
$$

(ii) For $\alpha \in(0,1)$ let

$$
I(\alpha)=\left(\int_{0}^{1} \frac{\left(1-x^{2}\right)^{\frac{n-1}{2}} d x}{(1-\alpha x)^{n+1}}\right)\left(\frac{\alpha^{\frac{n+1}{2}}(1-\alpha)^{\frac{n+1}{2}} n !}{2^{\frac{n-1}{2}}\left(\Gamma\left(\frac{n+1}{2}\right)\right)^{2}}\right) .
$$

Then

$$
I(\alpha) \leq 1 \quad \text { and } \quad \lim _{\alpha \rightarrow 1} I(\alpha)=1 .
$$

(iii) Let $a, b>0, n \in \mathbf{N}$ and $\lambda a<1$. Then

$$
\int_{-b}^{a} \frac{\left(1-\frac{y}{a}\right)^{n-1}}{(1-\lambda y)^{n+1}} d y=\frac{(a+b)^{n}}{n a^{n-1}(1-\lambda a)(1+\lambda b)^{n}} .
$$

Proof. (i) We put $x=\left(1-\frac{1-\alpha}{1+\alpha} u\right) /\left(1+\frac{1 \alpha}{1+\alpha} u\right)$. This gives (i).

(ii) Put $x=1-w \frac{1-\alpha}{\alpha}$. Then

$$
I(\alpha)=\frac{n !}{2^{\frac{n-1}{2}}\left(\Gamma\left(\frac{n+1}{2}\right)\right)^{2}} \int_{0}^{\frac{\alpha}{1-\alpha}} \frac{w^{\frac{n-1}{2}}\left(2-\frac{1-\alpha}{\alpha} w\right)^{\frac{n-1}{2}} d w}{(1+w)^{n+1}} .
$$

The upper estimate for (ii) follows immediately from this last expression. And by the monotone convergence theorem this last expression tends to

$$
\frac{n !}{\left(\Gamma\left(\frac{n+1}{2}\right)\right)^{2}} \int_{0}^{\infty} \frac{w^{\frac{n-1}{2}} d w}{(1+w)^{n+1}},
$$

which is equal to 1.

(iii) Note that

$$
\int \frac{\left(1-\frac{y}{a}\right)^{n-1}}{(1-\lambda y)^{n+1}} d y=\frac{\left(1-\frac{y}{a}\right)^{n}}{n\left(\lambda-\frac{1}{a}\right)(1-\lambda y)^{n}} .
$$

This immediately implies (iii). 
Corollary 5. Let $B(0, r)$ be the $n$-dimensional Euclidean ball with radius $r$ centered at 0 . For $u \in S^{n-1}$ let $x=\lambda u, 0 \leq \lambda<r$. Then

$$
\left|(B(0, r))^{x}\right|=\frac{v_{n}}{r^{n}\left(1-\left(\frac{\lambda}{r}\right)^{2}\right)^{\frac{n+1}{2}}} .
$$

Proof. The proof follows from (2) and Lemma 4 (i).

Next we estimate the "size" of $S(K, t)$ in terms of ellipsoids. Recall that for a convex body $K$ the Binet ellipsoid $E(K)$ is defined by (see [Mi-P])

$$
\|u\|_{E(K)}^{2}=\frac{1}{|K|} \int_{K}\langle x, u\rangle^{2} d x, \quad \text { for all } \mathrm{u} \in \mathbf{R}^{n} .
$$

We first treat the case when $K$ is a symmetric convex body.

Theorem 6. Let $K$ be a symmetric convex body in $\mathbf{R}^{n}$. For all $t \in \mathbf{R}$

$$
d_{n}(t) E\left(K^{0}\right) \subseteq S(K, t) \subseteq c_{n}(t) E\left(K^{0}\right),
$$

where

and

$$
d_{n}(t)=\frac{1}{\sqrt{3} n}\left(1-\frac{|K|\left|K^{0}\right|}{t v_{n}^{2}}\right)^{\frac{1}{2}}
$$

$c_{n}(t)=\min \left\{\left(\frac{2}{(n+1)(n+2)}\right)^{\frac{1}{2}}\left(\frac{t v_{n}^{2}}{|K|\left|K^{0}\right|}\right)^{\frac{1}{2}}\left(1-\frac{|K|\left|K^{0}\right|}{t v_{n}^{2}}\right)^{\frac{1}{2}}, \sqrt{2}\left(1-\left(\frac{|K|\left|K^{0}\right|}{t v_{n}^{2}}\right)^{\frac{1}{n}}\right)^{\frac{1}{2}}\right\}$.

Remarks. (i) In particular, for any ellipsoid $E, S(E, 1)=\{0\}$.

(ii) If $t \rightarrow \frac{|K|\left|K^{0}\right|}{v_{n}^{2}}$, then $S(K, t) \rightarrow\{0\}$.

(iii) The second expression in $c_{n}(t)$ gives a better estimate from above than the first iff $|K|\left|K^{0}\right| / t v_{n}^{2}$ is of a smaller order of magnitude than $(n \log n)^{-1}$.

(iv) Recall that for two isomorphic Banach spaces $E$ and $F$ the Banach-Mazur distance $d(E, F)$ is defined by

$$
d(E, F)=\inf \left\{\|T\|\left\|T^{-1}\right\|: T \text { is an isomorphism from } E \text { onto } F\right\} .
$$

For symmetric convex bodies $K, L$ in $\mathbf{R}^{n}$ we define

$$
d(K, L)=d\left(\left(\mathbf{R}^{n},\|\cdot\|_{K}\right),\left(\mathbf{R}^{n},\|\cdot\|_{L}\right)\right) .
$$

Then it follows from Theorem 6 that

$$
d\left(S(K, t), E\left(K^{0}\right)\right) \leq\left(\frac{6 t v_{n}^{2}}{|K|\left|K^{0}\right|}\right)^{\frac{1}{2}}
$$

Thus for $\rho \in \mathbf{R}, \rho>1$,

$$
d\left(\left\{x \in K:\left|K^{x}\right| \leq \rho\left|K^{0}\right|\right\}, E\left(K^{0}\right)\right) \leq(6 \rho)^{\frac{1}{2}},
$$

independent of $K$ and $n$. It follows that for fixed $\rho,\left\{x ;\left|K^{x}\right| \leq \rho\left|K^{0}\right|\right\}$ is almost an ellipsoid.

Proof of Theorem 6. Let $u \in S^{n-1}, \lambda \in \mathbf{R}, 0 \leq \lambda<\frac{1}{\|u\|_{K}}$ and $x=\lambda u$. By (2) and symmetry,

$$
\left|K^{x}\right|=\int_{0}^{\|u\|_{K}} \phi(y)\left(\frac{1}{(1-\lambda y)^{n+1}}+\frac{1}{(1+\lambda y)^{n+1}}\right) d y,
$$

where $\phi=\phi_{K^{0}}^{u}$. For fixed $\lambda \geq 0$ put

$$
f_{\lambda}(y)=\frac{1}{(1-\lambda y)^{n+1}}+\frac{1}{(1+\lambda y)^{n+1}} .
$$


Observe that $f_{\lambda}$ is increasing in $y$, if $y \geq 0$. Put

$$
a=\frac{n \int_{0}^{\infty} \phi(y) d y}{\phi(0)}=\frac{n\left|K^{0}\right|}{2 \phi(0)}
$$

Now we distinguish two cases.

1. $\lambda a<1$. Then we claim that for all functions $\psi: \mathbf{R}_{0}^{+} \rightarrow \mathbf{R}_{0}^{+}$such that $\psi^{\frac{1}{n-1}}$ is continuous on its support and continuous from the right at 0 , decreasing and concave on its support and such that

$$
\begin{gathered}
\text { (i) } \psi(0)=\phi(0), \\
\text { (ii) } \int_{0}^{\infty} \psi(y) d y=\int_{0}^{\infty} \phi(y) d y=\frac{\left|K^{0}\right|}{2},
\end{gathered}
$$

the integral $\int_{0}^{\infty} \psi(y) f_{\lambda}(y) d y$ is maximal if $\psi$ is of the form

$$
\psi_{0}(y)= \begin{cases}\phi(0)\left(1-\frac{y}{a}\right)^{n-1} & \text { if } y \in[0, a] \\ 0 & \text { otherwise. }\end{cases}
$$

Indeed, let $\psi$ be a function with above properties and with support on $[0, \tilde{a}]$. Put

$$
H(t)=\int_{t}^{a} \psi(y) d y-\int_{t}^{a} \psi_{0}(y) d y .
$$

Note that $\tilde{a} \leq a, H(0)=0=H(a)$ and that the derivative of $H$ with respect to $t$ is first negative, then positive; therefore $H(t) \leq 0$.

Consequently (with $g_{\lambda}(y)=f_{\lambda}(y)-2$ )

$$
\begin{gathered}
\int_{0}^{\infty} \psi(y) g_{\lambda}(y) d y=\int_{y=0}^{\infty} \psi(y)\left(\int_{t=0}^{y} g_{\lambda}^{\prime}(t) d t\right) d y=\int_{t=0}^{\infty} g_{\lambda}^{\prime}(t)\left(\int_{t}^{\infty} \psi(y) d y\right) d t \\
\leq \int_{0}^{\infty} g_{\lambda}^{\prime}(t)\left(\int_{t}^{\infty} \psi_{0}(y) d y\right) d t=\int_{0}^{\infty} \psi_{0}(y) g_{\lambda}(y) d y .
\end{gathered}
$$

From this the above claim follows.

Hence

$$
\left|K^{x}\right| \leq \phi(0) \int_{0}^{a}\left(\frac{\left(1-\frac{y}{a}\right)^{n-1}}{(1-\lambda y)^{n+1}}+\frac{\left(1-\frac{y}{a}\right)^{n-1}}{(1+\lambda y)^{n+1}}\right) d y=\frac{\left|K^{0}\right|}{1-\lambda^{2} a^{2}} .
$$

Here we have used Lemma 4 (iii).

For $x=\lambda u \in \partial S(K, t)$,

$$
1=\|x\|_{S(K, t)}=\lambda\|u\|_{S(K, t)}
$$

and

$$
t=\frac{|K|\left|K^{x}\right|}{v_{n}^{2}}
$$

Therefore

and hence by (3)

$$
t \leq \frac{|K|\left|K^{0}\right|}{v_{n}^{2}\left(1-\lambda^{2} a^{2}\right)}
$$

$$
\|u\|_{S(K, t)} \leq \frac{n}{2}\left(1-\frac{|K|\left|K^{0}\right|}{t v_{n}^{2}}\right)^{-\frac{1}{2}} \frac{\left|K^{0}\right|}{\phi(0)} .
$$

Now ([B], respectively $[\mathrm{He}]$; see also $[\mathrm{Mi}-\mathrm{P}])$

$$
\frac{\left|K^{0}\right|}{\phi(0)} \leq 2 \sqrt{3}\left(\frac{\int_{K^{0}}|\langle x, u\rangle|^{2} d x}{\left|K^{0}\right|}\right)^{\frac{1}{2}}=2 \sqrt{3}\|u\|_{E\left(K^{0}\right)},
$$


and thus

$$
S(K, t) \supseteq \frac{1}{\sqrt{3} n}\left(1-\frac{|K|\left|K^{0}\right|}{t v_{n}^{2}}\right)^{\frac{1}{2}} E\left(K^{0}\right) .
$$

2. $\lambda a \geq 1$. Again let $x=\lambda u \in \partial S(K, t)$. By definition of $a,(3)$ and (5)

$$
\|u\|_{S(K, t)} \leq \sqrt{3} n\|u\|_{E\left(K^{0}\right)}
$$

This implies that

$$
S(K, t) \supseteq \frac{1}{\sqrt{3} n} E\left(K^{0}\right),
$$

which proves the inclusion from below also in this case.

On the other hand, by (1) and symmetry

$$
\begin{gathered}
\left|K^{x}\right|=\frac{1}{2 n} \int_{S^{n-1}}\left(\frac{1}{\left(\|v\|_{K^{0}}-\langle v, x\rangle\right)^{n}}+\frac{1}{\left(\|v\|_{K^{0}}+\langle v, x\rangle\right)^{n}}\right) d \sigma(v) \\
\geq \frac{1}{2 n} \int_{S^{n-1}}\left(2+n(n+1)\left(\frac{\langle x, v\rangle}{\|v\|_{K^{0}}}\right)^{2}\right) \frac{d \sigma(v)}{\|v\|_{K^{0}}^{n}} \\
\quad=\left|K^{0}\right|+\frac{(n+1)(n+2)}{2} \int_{K^{0}}|\langle x, y\rangle|^{2} d y \\
=\left|K^{0}\right|+\frac{(n+1)(n+2)}{2} \lambda^{2}\left|K^{0}\right|\|u\|_{E\left(K^{0}\right)}^{2} .
\end{gathered}
$$

Then (3) and (4) give

$$
\|u\|_{S(K, t)} \geq \frac{((n+1)(n+2))^{\frac{1}{2}}}{\sqrt{2}}\left(\frac{t v_{n}^{2}}{|K|\left|K^{0}\right|}-1\right)^{-\frac{1}{2}}\|u\|_{E\left(K^{0}\right)},
$$

or, equivalently,

$$
S(K, t) \subseteq \frac{\sqrt{2}}{((n+1)(n+2))^{\frac{1}{2}}}\left(\frac{t v_{n}^{2}}{|K|\left|K^{0}\right|}-1\right)^{\frac{1}{2}} E\left(K^{0}\right) .
$$

Using (2) and a minimality argument similar to the maximality argument of the above claim, we get the other upper bound. Namely, for fixed $\lambda$ and for all functions $\psi: \mathbf{R}_{0}^{+} \rightarrow \mathbf{R}_{0}^{+}$such that $\psi^{\frac{1}{n-1}}$ is continuous on its support and continuous from the right at 0 , decreasing and concave on its support and such that

$$
\text { (i) } \psi(0)=\phi(0) \text {, }
$$

$$
\text { (ii) } \int_{0}^{\infty} \psi(y) d y=\int_{0}^{\infty} \phi(y) d y=\frac{\left|K^{0}\right|}{2},
$$

the integral $\int_{0}^{\infty} \psi(y) f_{\lambda}(y) d y$ is minimal if $\psi$ is of the form

$$
\psi(y)= \begin{cases}\phi(0) & \text { if } y \in[0, a] \\ 0 & \text { otherwise }\end{cases}
$$

where

$$
a=\frac{\left|K^{0}\right|}{2 \phi(0)} .
$$

Note that in this situation $\lambda a<1$ always.

Consequently

$$
\left|K^{x}\right| \geq \phi(0) \int_{0}^{a} f_{\lambda}(y) d y \geq \frac{\left|K^{0}\right|}{\left(1-\lambda^{2} a^{2}\right)^{n}} .
$$


Then we again use (3), (4) and the fact that ([B], or respectively [He]; see also $[\mathrm{Mi}-\mathrm{P}])$

$$
\frac{\left|K^{0}\right|}{\phi(0)} \geq \sqrt{2}|| u \|_{E\left(K^{0}\right)}
$$

and get

$$
S(K, t) \subseteq \sqrt{2}\left(1-\left(\frac{|K|\left|K^{0}\right|}{t v_{n}^{2}}\right)^{\frac{1}{n}}\right)^{\frac{1}{2}} E\left(K^{0}\right) .
$$

Now we consider the non-symmetric case.

Theorem 7. Let $K$ be a convex body in $\mathbf{R}^{n}$. Then

$$
d_{n}^{\prime}(t) E\left(K^{0}\right) \subseteq S(K, t) \subseteq c_{n}^{\prime}(t) E\left(K^{0}\right),
$$

where

$$
c_{n}^{\prime}(t)=\frac{2 \sqrt{2}}{((e-2)(n+1)(n+2))^{\frac{1}{2}}}\left(\frac{t v_{n}^{2}}{|K|\left|K^{0}\right|}\right)^{\frac{1}{2}}\left(1-\frac{|K|\left|K^{0}\right|}{t v_{n}^{2}}\right)^{\frac{1}{2}}
$$

and

$$
d_{n}^{\prime}(t)=d_{n}(t)=\frac{1}{\sqrt{3} n}\left(1-\frac{|K|\left|K^{0}\right|}{t v_{n}^{2}}\right)^{\frac{1}{2}} .
$$

Proof. By (2) we get for $u \in S^{n-1}$ and $x=\lambda u$ with $0 \leq \lambda<1 / h_{K^{0}}(u)$ that

$$
\left|K^{x}\right|=\int_{-h_{K^{0}}(-u)}^{h_{K^{0}}(u)} \frac{\phi(y)}{(1-\lambda y)^{n+1}} d y,
$$

where $\phi=\phi_{K^{0}}^{u}$.

Notice that $K^{0}$ has its center of gravity at 0 , as $K$ has its Santaló-point at 0 . Therefore

$$
\int_{-h_{K^{0}}(-u)}^{h_{K^{0}}(u)} y \phi(y) d y=0
$$

Notice also that

$$
1=h_{S(K, t)^{0}}(x)=\lambda h_{S(K, t)^{0}}(u) .
$$

Now we apply the following result of Fradelizi $[\mathrm{F}]$ to the functions $\phi(y)$ and $f_{\lambda}(y)=\frac{1}{(1-\lambda y)^{n+1}}$ to get the same upper estimate for $\left|K^{x}\right|$ as in the proof of Theorem 6. Therefore $d_{n}^{\prime}(t)=d_{n}(t)$.

Theorem $([\mathrm{F}])$. Let $\psi: \mathbf{R} \rightarrow \mathbf{R}, \psi \geq 0$, be such that $\psi^{\frac{1}{n-1}}$ is continuous and concave on its support and such that $\int_{-\infty}^{\infty} y \psi(y) d y=0$. Let $f: \mathbf{R} \rightarrow \mathbf{R}$ be any convex function. Then, if

$$
a=\frac{n \int_{-\infty}^{\infty} \psi(y) d y}{2 \psi(0)}
$$

one has

$$
\int_{-\infty}^{\infty} \psi(y) f(y) d y \leq \psi(0) \int_{-a}^{a}\left(1-\frac{|y|}{a}\right)^{n-1} f(y) d y
$$


For the right-hand inclusion write

$$
\begin{aligned}
\left|K^{x}\right|= & \int_{-h_{K^{0}}(-u)}^{0} \frac{\phi(y)}{(1-\lambda y)^{n+1}} d y+\int_{0}^{h_{K^{0}}(u)} \frac{\phi(y)}{(1-\lambda y)^{n+1}} d y \\
\geq & \int_{-h_{K^{0}}(-u)}^{0} \phi(y)(1+(n+1) \lambda y) d y \\
& \quad+\int_{0}^{h_{K^{0}}(u)} \phi(y)\left(1+(n+1) \lambda y+\frac{(n+1)(n+2)}{2} \lambda^{2} y^{2}\right) d y \\
= & \left|K^{0}\right|+\frac{(n+1)(n+2)}{2} \lambda^{2} \int_{0}^{h_{K^{0}}(u)} y^{2} \phi(y) d y,
\end{aligned}
$$

where for the last equality we have used the fact that the center of gravity is at 0 . Let $a$ be such that

$$
\int_{0}^{h_{K^{0}}(u)} y \phi(y) d y=\phi(0) \frac{a^{2}}{n(n+1)}=\int_{-a}^{\frac{a}{n}} y \psi_{0}(y) d y
$$

where $\psi_{0}(y)=\phi(0)\left(1+\frac{y}{a}\right)^{n-1}$.

Now one shows as in the beginning of the proof of Theorem 6 that for all functions $\psi: \mathbf{R}_{0}^{+} \rightarrow \mathbf{R}_{0}^{+}$such that $\psi^{\frac{1}{n-1}}$ is continuous on its support and continuous from the right at 0 , concave on its support and such that

$$
\begin{aligned}
(\text { i) } \quad \psi(0) & =\phi(0), \\
\text { (ii) } \quad \int_{0}^{\infty} y \psi(y) d y & =\int_{0}^{\infty} y \phi(y) d y,
\end{aligned}
$$

the integral $\int_{0}^{\infty} y^{2} \psi(y) d y$ is minimal if $\psi$ is of the form

$$
\psi_{0}(y)= \begin{cases}\phi(0)\left(1+\frac{y}{a}\right)^{n-1} & \text { if } y \in\left[0, \frac{a}{n}\right] \\ 0 & \text { otherwise }\end{cases}
$$

and that for all functions $\psi: \mathbf{R}_{0}^{-} \rightarrow \mathbf{R}_{0}^{+}$such that $\psi^{\frac{1}{n-1}}$ is continuous on its support and continuous from the left at 0 , concave on its support and such that

(i) $\psi(0)=\phi(0)$,

$$
\text { (ii) } \int_{-\infty}^{0} y \psi(y) d y=\int_{-\infty}^{0} y \phi(y) d y
$$

the integral $\int_{-\infty}^{0} y^{2} \psi(y) d y$ is maximal if $\psi$ is of the form

$$
\psi_{0}(y)= \begin{cases}\phi(0)\left(1+\frac{y}{a}\right)^{n-1} & \text { if } y \in[-a, 0] \\ 0 & \text { otherwise. }\end{cases}
$$

Therefore

$$
\int_{0}^{h_{K^{0}}(u)} y^{2} \phi(y) d y \geq \int_{0}^{\frac{a}{n}} y^{2} \psi_{0}(y) d y=\frac{\left(1+\frac{1}{n}\right)^{n+1}-2}{n(n+1)(n+2)} a^{3} \phi(0)
$$

and

$$
\int_{-h_{K^{0}}(-u)}^{0} y^{2} \phi(y) d y \leq \int_{-a}^{0} y^{2} \psi_{0}(y) d y=\frac{2}{n(n+1)(n+2)} a^{3} \phi(0) .
$$

Thus we get

$$
\int_{0}^{h_{K^{0}}(u)} y^{2} \phi(y) d y \geq \frac{e-2}{2} \int_{-h_{K^{0}}(-u)}^{0} y^{2} \phi(y) d y
$$


and therefore

$$
\int_{0}^{h_{K^{0}}(u)} y^{2} \phi(y) d y \geq \frac{e-2}{4} \int_{-h_{K^{0}}(-u)}^{h_{K^{0}}(u)} y^{2} \phi(y) d y .
$$

It follows that

$$
\left|K^{x}\right| \geq\left|K^{0}\right|\left(1+\frac{(n+1)(n+2)(e-2)}{8} \lambda^{2}\|u\|_{E\left(K^{0}\right)}^{2}\right),
$$

which implies, using (6),

$$
S(K, t) \subseteq \frac{2 \sqrt{2}}{((e-2)(n+1)(n+2))^{\frac{1}{2}}}\left(\frac{t v_{n}^{2}}{|K|\left|K^{0}\right|}\right)^{\frac{1}{2}}\left(1-\frac{|K|\left|K^{0}\right|}{t v_{n}^{2}}\right)^{\frac{1}{2}} E\left(K^{0}\right) .
$$

Next we estimate the "size" of $S(K, t)$ in terms of the body $K$. We need the following lemma.

Lemma 8 (see for instance [S2]). Let $K$ be a convex body in $\mathbf{R}^{n}$ with center of gravity at 0 . Then

$$
\frac{1}{e} \leq \frac{1}{|K|} \int_{0}^{h_{K}(u)} \phi(y) d y \leq 1-\frac{1}{e}
$$

Theorem 9. Let $K$ be a convex body in $\mathbf{R}^{n}$. Then:

(i)

$$
\left(1-\left(\frac{|K|\left|K^{0}\right|}{t v_{n}^{2}}\right)^{\frac{1}{n}}\right) K \subseteq S(K, t) \subseteq\left(1-\frac{|K|\left|K^{0}\right|}{e t v_{n}^{2}}\right) K .
$$

(ii) If in addition $K$ is symmetric, then

$$
\left(1-\left(\frac{|K|\left|K^{0}\right|}{t v_{n}^{2}}\right)^{\frac{1}{n}}\right) K \subseteq S(K, t) \subseteq\left(1-\frac{|K|\left|K^{0}\right|}{t v_{n}^{2}}\right)^{\frac{1}{2}} K .
$$

Proof. Let $u \in S^{n-1}, \lambda \in \mathbf{R}, 0 \leq \lambda \leq 1$, be given and let $x=\frac{\lambda}{h_{K^{0}}(u)} u$. Then $K$ contains $\alpha K+x$ for all $\alpha, 0 \leq \alpha \leq 1-\lambda$, and consequently $K^{x} \subseteq \frac{1}{\alpha} K^{0}$; therefore for $x \in \partial S(K, t)$ we have

$$
t=\frac{|K|\left|K^{x}\right|}{v_{n}^{2}} \leq \frac{|K|\left|K^{0}\right|}{\alpha^{n} v_{n}^{2}}
$$

and hence

$$
\alpha \leq\left(\frac{|K|\left|K_{0}\right|}{t v_{n}^{2}}\right)^{\frac{1}{n}} .
$$

Thus for all $\lambda$ with $\lambda \leq 1-\left(|K|\left|K_{0}\right| / t v_{n}^{2}\right)^{\frac{1}{n}}$ we have

$$
\frac{\lambda}{h_{K^{0}}(u)} u \in S(K, t) \text {. }
$$

This proves the left-hand inclusion.

For the right-hand inclusion we first treat the symmetric case.

Let $x=\lambda u, u \in S^{n-1}, 0 \leq \lambda<\|u\|_{K}^{-1}$. Let $f_{\lambda}$ be as in the proof of Theorem 6 . By (2) and symmetry,

$$
\left|K^{x}\right|=\int_{0}^{\|u\|_{K}} \phi(y) f_{\lambda}(y) d y
$$


Notice that for all functions $\psi: \mathbf{R}_{0}^{+} \rightarrow \mathbf{R}_{0}^{+}$such that $\psi^{\frac{1}{n-1}}$ is continuous on its support and continuous at 0 from the right, decreasing and concave on its support and such that

$$
\begin{gathered}
\text { (i) } \psi>0 \text { on }\left[0,\|u\|_{K}\right), \quad \psi=0 \text { on } \quad\left[\|u\|_{K}, \infty\right), \\
\text { (ii) } \int_{0}^{\|u\|_{K}} \psi(y) d y=\int_{0}^{\|u\|_{K}} \phi(y) d y=\frac{\left|K^{0}\right|}{2},
\end{gathered}
$$

the integral $\int_{0}^{\|u\|_{K}} \psi(y) f_{\lambda}(y) d y$ is smallest if $\psi$ is of the form

$$
\psi(y)= \begin{cases}c\left(1-\frac{y}{\|u\|_{K}}\right)^{n-1} & \text { if } y \in\left[0,\|u\|_{K}\right), \\ 0 & \text { otherwise }\end{cases}
$$

where

Hence

$$
c=\frac{n\left|K^{0}\right|}{2|| u||_{K}}
$$

$$
\begin{gathered}
\left|K^{x}\right| \geq c \int_{0}^{\|u\|_{K}}\left(\frac{\left(1-\frac{y}{\|u\|_{K}}\right)^{n-1}}{(1-\lambda y)^{n+1}}+\frac{\left(1-\frac{y}{\|u\|_{K}}\right)^{n-1}}{(1+\lambda y)^{n+1}}\right) d y \\
=\frac{\left|K^{0}\right|}{1-\lambda^{2}\|u\|_{K}^{2}},
\end{gathered}
$$

which implies

$$
S(K, t) \subseteq\left(1-\frac{|K|\left|K_{0}\right|}{t v_{n}^{2}}\right)^{\frac{1}{2}} K .
$$

Next we consider the non-symmetric case:

$$
\begin{aligned}
\left|K^{x}\right|=\int_{0}^{h_{K^{0}}(u)} & \frac{\phi(y)}{(1-\lambda y)^{n+1}} d y+\int_{0}^{h_{K^{0}}(-u)} \frac{\phi(-y)}{(1+\lambda y)^{n+1}} d y \\
& \geq \int_{0}^{h_{K^{0}}(u)} \frac{\phi(y)}{(1-\lambda y)^{n+1}} d y .
\end{aligned}
$$

Fix $\lambda$, and note again that among all functions $\psi: \mathbf{R}_{0}^{+} \rightarrow \mathbf{R}_{0}^{+}$such that $\psi^{\frac{1}{n-1}}$ is continuous on its support and continuous from the right at 0 , decreasing and concave on its support and for which

$$
\begin{gathered}
\psi>0 \text { on }\left[0, h_{K^{0}}(u)\right), \quad \psi=0 \text { on }\left[h_{K^{0}}(u), \infty\right), \\
\int_{0}^{h_{K^{0}}(u)} \psi(y) d y=\int_{0}^{h_{K^{0}}(u)} \phi(y) d y,
\end{gathered}
$$

the integral $\int_{0}^{h_{K^{0}}(u)} \frac{\psi(y)}{(1-\lambda y)^{n+1}} d y$ is smallest if $\psi$ is of the form

$$
\psi(y)= \begin{cases}c\left(1-\frac{y}{h_{K^{0}}(u)}\right)^{n-1} & \text { if } y \in\left[0, h_{K^{0}}(u)\right), \\ 0 & \text { otherwise }\end{cases}
$$

where

$$
c=\frac{n \int_{0}^{h_{K^{0}}(u)} \phi(y) d y}{h_{K^{0}}(u)} .
$$

Arguments similar to the previous ones, together with Lemma 8, then finish the proof. 


\section{SANTALÓ-REgIONS AND AFFINE SURFACE AREA}

Recall that for a convex body $K$ in $\mathbf{R}^{n}$ the affine surface area is

$$
a s(K)=\int_{\partial K} \kappa(x)^{\frac{1}{n+1}} d \mu(x),
$$

where $\kappa(x)$ is the (generalized) Gaussian curvature in $x \in \partial K$ and $\mu$ is the surface measure on $\partial K$. We prove here

Theorem 10. Let $K$ be a convex body in $\mathbf{R}^{n}$. Then

$$
\lim _{t \rightarrow \infty} t^{\frac{2}{n+1}}(|K|-|S(K, t)|)=\frac{1}{2}\left(\frac{|K|}{v_{n}}\right)^{\frac{2}{n+1}} \operatorname{as}(K) .
$$

In the proof of Theorem 10 we follow the ideas of $[\mathrm{S}-\mathrm{W}]$. We need several lemmas for the proof. We also use the following notations. For $x \in \partial K, N(x)$ is the outer unit normal vector to $\partial K$ in $x$. For two points $x$ and $y$ in $\mathbf{R}^{n},[x, y]=\{\alpha x+(1-\alpha) y$ : $0 \leq \alpha \leq 1\}$ denotes the line segment from $x$ to $y$.

The proof of the following lemma is standard.

Lemma 11. Let $K$ and $L$ be two convex bodies in $\mathbf{R}^{n}$ such that $0 \in \operatorname{int}(L)$ and $L \subseteq K$. Then

$$
|K|-|L|=\frac{1}{n} \int_{\partial K}\langle x, N(x)\rangle\left(1-\left(\frac{\left\|x_{L}\right\|}{\|x\|}\right)^{n}\right) d \mu(x),
$$

where $x_{L}=[0, x] \cap \partial L$.

For $x \in \partial K$ denote by $r(x)$ the radius of the biggest Euclidean ball contained in $K$ that touches $\partial K$ at $x$. More precisely,

$$
r(x)=\max \{r: x \in B(y, r) \subset K \text { for some } \mathrm{y} \in K\} .
$$

Remark. It was shown in $[\mathrm{S}-\mathrm{W}]$ that

(i) if $B(0,1) \subset K$, then

$$
\mu\{x \in \partial K: r(x) \geq \beta\} \geq(1-\beta)^{n-1} \operatorname{vol}_{n-1}(\partial K),
$$

(ii)

$$
\int_{\partial K} r(x)^{-\alpha} d \mu(x)<\infty \quad \text { for all } \quad \alpha, \quad 0<\alpha<1
$$

We postpone the proof of the next two lemmas, which we use for the proof of Theorem 10.

Lemma 12. Suppose 0 is in the interior of $K$. Then, for all $x$ with $r(x)>0$ and for all $t$ such that $(S(K, t)$ has non-empty interior, we have

$$
0 \leq \frac{1}{n}\langle x, N(x)\rangle t^{\frac{2}{n+1}}\left(1-\left(\frac{\left\|x_{t}\right\|}{\|x\|}\right)^{n}\right) \leq c r(x)^{-\frac{n-1}{n+1}},
$$

where $x_{t}=[0, x] \cap \partial S(K, t)$ and $c$ is a constant independent of $x$ and $t$.

Lemma 13. Suppose 0 is in the interior of $K$. Then the limit

$$
\lim _{t \rightarrow \infty} \frac{1}{n}\langle x, N(x)\rangle t^{\frac{2}{n+1}}\left(1-\left(\frac{\left\|x_{t}\right\|}{\|x\|}\right)^{n}\right)
$$


exists a.e. and is equal to

$$
\frac{1}{2}\left(\frac{|K|}{v_{n}}\right)^{\frac{2}{n+1}} \kappa(x)^{\frac{1}{n+1}}
$$

where $\kappa(x)$ is the Gaussian curvature at $x \in \partial K$.

Proof of Theorem 10. We may assume that 0 is in the interior of $K$. By Lemma 11 and with the notation of Lemma 12 we have

$$
|K|-|S(K, t)|=\frac{1}{n} \int_{\partial K}\langle x, N(x)\rangle\left(1-\left(\frac{\left\|x_{t}\right\|}{\|x\|}\right)^{n}\right) d \mu(x) .
$$

By Lemma 12 and the remark preceding it, the functions under the integral sign are bounded uniformly in $t$ by an $L^{1}$-function, and by Lemma 13 they converge pointwise a.e. We apply Lebesgue's convergence theorem.

Proof of Lemma 12. Let $x \in \partial K$ be such that $r(x)>0$. As $\left\|x_{t}\right\|=\|x\|-\left\|x-x_{t}\right\|$, we have

$$
\frac{1}{n}\langle x, N(x)\rangle\left(1-\left(\frac{\left\|x_{t}\right\|}{\|x\|}\right)^{n}\right) \leq\left\langle\frac{x}{\|x\|}, N(x)\right\rangle\left\|x-x_{t}\right\| .
$$

a) We consider first the case where

$$
\left\|x-x_{t}\right\|<r(x)\left\langle\frac{x}{\|x\|}, N(x)\right\rangle .
$$

Let $\tilde{\rho}=\left\|x_{t}-(x-r(x) N(x))\right\|$. By assumption $0<\tilde{\rho}<r(x)$. Computing $\tilde{\rho}$, we get

$$
\tilde{\rho}=\left(\left\|x-x_{t}\right\|^{2}+r(x)^{2}-2 r(x)\left\|x-x_{t}\right\|\left\langle\frac{x}{\|x\|}, N(x)\right\rangle\right)^{1 / 2} .
$$

Since $K$ contains the Euclidean ball of radius $r(x)$ centered at $x-r(x) N(x), K^{x_{t}}$ is contained in the polar (with respect to $x_{t}$ ) of the Euclidean ball with radius $r(x)$. Hence by Corollary 5 ,

$$
t=\frac{|K|\left|K^{x_{t}}\right|}{v_{n}^{2}} \leq \frac{|K|}{v_{n} r(x)^{n}\left(1-\left(\frac{\tilde{\rho}}{r(x)}\right)^{2}\right)^{\frac{n+1}{2}}},
$$

and therefore, using (7),

$$
\frac{1}{n}\langle x, N(x)\rangle t^{\frac{2}{n+1}}\left(1-\left(\frac{\left\|x_{t}\right\|}{\|x\|}\right)^{n}\right) \leq\left(\frac{|K|}{v_{n}}\right)^{\frac{2}{n+1}} r(x)^{-\frac{n-1}{n+1}},
$$

which proves Lemma 12 in this case.

b) Now we consider the case where

$$
\left\|x-x_{t}\right\| \geq r(x)\left\langle\frac{x}{\|x\|}, N(x)\right\rangle .
$$

We can suppose that $t$ is big enough so that $x_{t} \neq 0$. We choose $\alpha>0$ such that $B(0, \alpha) \subseteq K \subseteq B\left(0, \frac{1}{\alpha}\right)$ and $t$ so big that $x_{t} \notin B(0, \alpha)$. $K$ contains the spherical cone $C=\operatorname{co}[x, H \cap B(0, \alpha)]$, where $H$ is the hyperplane through 0 orthogonal to the line segment $[0, x]$. We get

Consequently

$$
\left|C^{x_{t}}\right|=\frac{v_{n-1}\|x\|^{n}}{n \alpha^{n-1}|| x_{t} \|\left(\|x\|-\left\|x_{t}\right\|\right)^{n}} .
$$

$$
t=\frac{|K|\left|K^{x_{t}}\right|}{v_{n}^{2}} \leq \frac{|K| v_{n-1}\|x\|^{n}}{n v_{n}^{2} \alpha^{n-1}\left\|x_{t}\right\|\left\|x-x_{t}\right\|^{n}}
$$


and hence, using (7),

$$
\frac{1}{n}\langle x, N(x)\rangle t^{\frac{2}{n+1}}\left(1-\left(\frac{\left\|x_{t}\right\|}{\|x\|}\right)^{n}\right) \leq\left(\frac{|K| v_{n-1}}{n v_{n}^{2}}\right)^{\frac{2}{n+1}} r(x)^{-\frac{n-1}{n+1}} \frac{1}{\alpha^{\frac{4 n}{n+1}}} .
$$

Proof of Lemma 13. As in the proof of Lemma 12, we can choose an $\alpha>0$ such that

Therefore

$$
B(0, \alpha) \subseteq K \subseteq B\left(0, \frac{1}{\alpha}\right)
$$

$$
1 \geq\left\langle\frac{x}{\|x\|}, N(x)\right\rangle \geq \alpha^{2} .
$$

Since $x$ and $x_{t}$ are collinear,

$$
\|x\|=\left\|x_{t}\right\|+\left\|x-x_{t}\right\|
$$

and hence

$$
\begin{gathered}
\frac{1}{n}\langle x, N(x)\rangle\left(1-\left(\frac{\left\|x_{t}\right\|}{\|x\|}\right)^{n}\right)=\frac{1}{n}\langle x, N(x)\rangle\left(\left(1-\left(1-\frac{\left\|x-x_{t}\right\|}{\|x\|}\right)^{n}\right)\right. \\
\geq\left\langle\frac{x}{\|x\|}, N(x)\right\rangle\left\|x-x_{t}\right\|\left(1-d \cdot \frac{\left\|x-x_{t}\right\|}{\|x\|}\right),
\end{gathered}
$$

for some constant $d$, if we choose $t$ sufficiently large. We denote by $\theta$ the angle between $x$ and $N(x)$. Then $\langle x /\|x\|, N(x)\rangle=\cos \theta$.

By [S-W], $r(x)>0$ a.e., and by [L2] the Dupin indicatrix exists a.e. and is an elliptic cylinder or an ellipsoid.

(i) Case where the indicatrix is an ellipsoid. This case can be reduced to the case of a sphere by an affine transformation with determinant 1 (see for instance $[\mathrm{S}-\mathrm{W}])$. Let $\sqrt{\rho(x)}$ be the radius of this sphere. Recall that we have to show that

$$
\lim _{t \rightarrow \infty} \frac{1}{n}\langle x, N(x)\rangle t^{\frac{2}{n+1}}\left(1-\left(\frac{\left\|x_{t}\right\|}{\|x\|}\right)^{n}\right)=\frac{1}{2}\left(\frac{|K|}{v_{n}}\right)^{\frac{2}{n+1}} \rho(x)^{-\frac{n-1}{n+1}} .
$$

We put $\rho(x)=\rho$ and we introduce a coordinate system such that $x=0$ and $N(x)=$ $(0, \ldots 0,-1) . H_{0}$ is the tangent hyperplane to $\partial K$ at $x=0$, and $\left\{H_{s}: s \geq 0\right\}$ is the family of hyperplanes parallel to $H_{0}$ that have non-empty intersection with $K$ and are at distance $s$ from $H_{0}$. For $s>0, H_{s}^{+}$is the halfspace generated by $H_{s}$ that contains $x=0$. For $a \in \mathbf{R}$, let $z_{a}=(0, \ldots, 0, a)$, and let $B_{a}=B\left(z_{a}, a\right)$ be the Euclidean ball with center $z_{a}$ and radius $a$. As in [W], for $\varepsilon>0$ we can choose $s_{0}$ so small that for all $s \leq s_{0}$

$$
B_{\rho-\varepsilon} \cap H_{s}^{+} \subseteq K \cap H_{s}^{+} \subseteq B_{\rho+\varepsilon} \cap H_{s}^{+} .
$$

For $\lambda \in \mathbf{R}$ let $G_{\lambda}=\left\{x:\left\langle x, z_{\rho+\varepsilon}-x_{t}\right\rangle=\lambda\right\}$ be a hyperplane orthogonal to the line segment $\left[x_{t}, z_{\rho+\varepsilon}\right]$, if $t$ is sufficiently large. Let $\lambda_{0}=\max \left\{\lambda: G_{\lambda}^{+} \cap B_{\rho+\varepsilon} \subseteq\right.$ $\left.H_{s_{0}}^{+} \cap B_{\rho+\varepsilon}\right\}$. Define $C$ to be the cone tangent to $B_{\rho+\varepsilon}$ at $G_{\lambda_{0}} \cap B_{\rho+\varepsilon}$, and choose the minimal $\lambda_{1}$ so that

$$
K \cap\left\{x: \lambda_{0} \leq\left\langle x, z_{\rho+\varepsilon}-x_{t}\right\rangle \leq \lambda_{1}\right\} \subseteq D=C \cap\left\{x: \lambda_{0} \leq\left\langle x, z_{\rho+\varepsilon}-x_{t}\right\rangle \leq \lambda_{1}\right\} .
$$

Then $K$ is contained in the union of the truncated cone $D$ of height $h=\left|\lambda_{1}-\lambda_{0}\right|$ and the cap $L=\left\{x \in B_{\rho+\varepsilon}:\left\langle x, z_{\rho+\varepsilon}-x_{t}\right\rangle \leq \lambda_{0}\right\}$ (see Figure 1). 


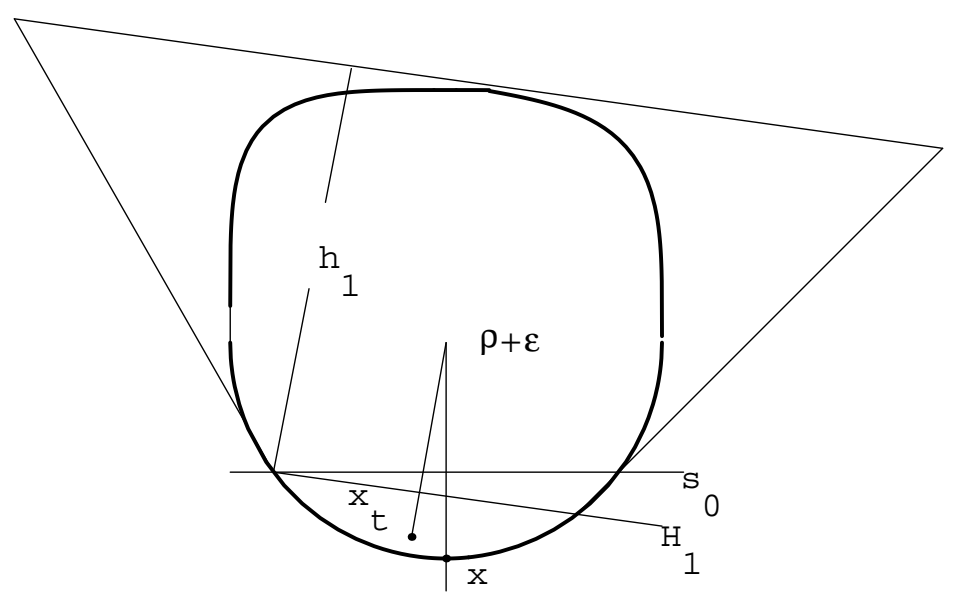

Figure 1. The estimate from below

Therefore

$$
K^{x_{t}} \supseteq(D \cup L)^{x_{t}}
$$

and to estimate $\left|K^{x_{t}}\right|$ we have to compute $\left|(D \cup L)^{x_{t}}\right|$. To do so we prove the following more general result.

Claim 1. Let $M$ be the convex body that is the union of a truncated spherical cone $D$ with height $h$ and a cap $L$ of a Euclidean ball with radius $r$ such that $D$ is "tangent" to $L$. For a point $x$ in $L$ and on the axis of symmetry of $M$ let a=distance $(x, D)$, $b=\operatorname{distance}(x, \partial L)$ and $b_{0}=\frac{r b+(a+b)(r-b)}{r-(a+b)}$ (see Figure 2). Then, if $x$ is such that $r>a+b$,

$$
\begin{gathered}
\left|M^{x}\right|=v_{n-1}\left(\frac{1}{r^{n}} \int_{\frac{r}{(r-b)+b_{0}}}^{1} \frac{\left(1-y^{2}\right)^{\frac{n-1}{2}}}{\left(1-\frac{(r-b) y}{r}\right)^{n+1}} d y\right. \\
\left.+\frac{1}{n}\left(\frac{1}{b_{0}}+\frac{1}{a+h}\right)\left(\frac{\left(2 r(a+b)-(a+b)^{2}\right)^{\frac{1}{2}}}{r b+(a+b)(r-b)}\right)^{n-1}\right) .
\end{gathered}
$$

Proof of Claim 1. We introduce a coordinate system such that $x=0$ and the $x_{1^{-}}$ axis coincides with the axis of symmetry of $M$ (see Figure 2).

Notice now that $M^{0}$ is such that each $(n-1)$-dimensional section orthogonal to the $x_{1}$-axis is an $(n-1)$-dimensional Euclidean ball with radius $l\left(x_{1}\right)$, where

$$
\begin{gathered}
l\left(x_{1}\right)=\frac{\left((a+h) x_{1}+1\right)\left(2 r(a+b)-(a+b)^{2}\right)^{\frac{1}{2}}}{\left(a+h+b_{0}\right)(r-(a+b))}, \quad \text { if } \quad-\frac{1}{a+h} \leq x_{1} \leq \frac{1}{b_{0}}, \\
l\left(x_{1}\right)=\frac{1}{r}\left(\left(1+x_{1}(r-b)\right)^{2}-r^{2} x_{1}^{2}\right)^{\frac{1}{2}}, \quad \text { if } \quad \frac{1}{b_{0}} \leq x_{1} \leq \frac{1}{b} .
\end{gathered}
$$

From this Claim 1 follows.

Now we apply Claim 1 to our situation. Then

$$
r=\rho+\varepsilon \text { and } b=\rho+\varepsilon-c,
$$

where

$$
c^{2}=\left\|x-x_{t}\right\|^{2}+(\rho+\varepsilon)^{2}-2(\rho+\varepsilon)\left\|x-x_{t}\right\| \cos \theta,
$$




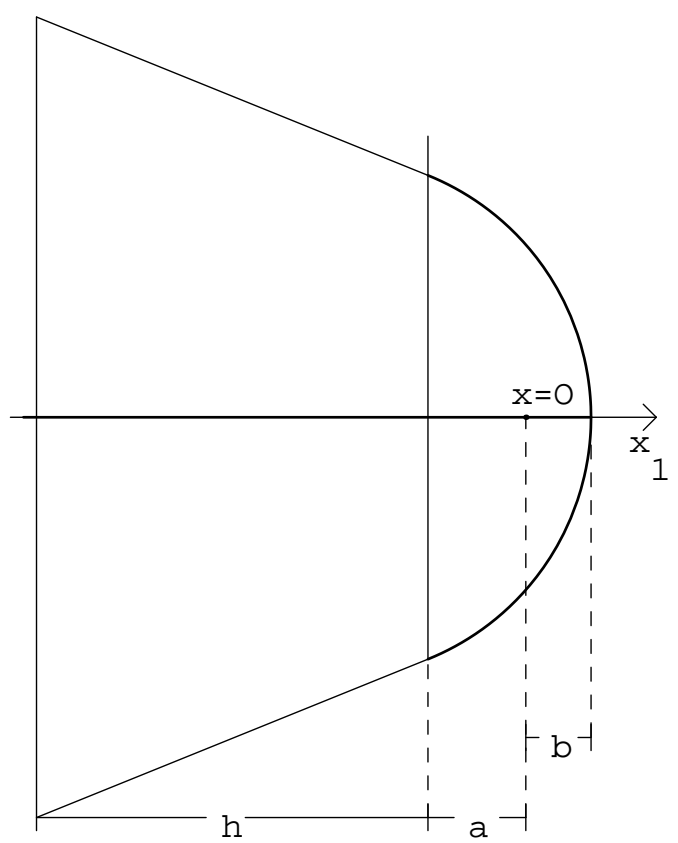

Figure 2. Claim 1

$$
a=c-\frac{\rho+\varepsilon-s_{0}}{c}\left(c^{2}-\left\|x-x_{t}\right\|^{2} \sin ^{2} \theta\right)^{\frac{1}{2}}-\frac{\left(2(\rho+\varepsilon) s_{0}-s_{0}^{2}\right)^{\frac{1}{2}}\left\|x-x_{t}\right\| \sin \theta}{c},
$$

and

$$
b_{0}=\frac{(\rho+\varepsilon)^{2}}{c-a}-c
$$

Therefore

$$
\begin{gathered}
\left|K^{x_{t}}\right| \geq \frac{v_{n-1}}{(\rho+\varepsilon)^{n}} \int_{0}^{1} \frac{\left(1-y^{2}\right)^{\frac{n-1}{2}}}{\left(1-\frac{c y}{\rho+\varepsilon}\right)^{n+1}} d y \\
+\frac{1}{n}\left(\frac{1}{b_{0}}+\frac{1}{a+h}\right) \frac{v_{n-1}}{r_{D}^{n-1}}-\frac{v_{n-1}}{(\rho+\varepsilon)^{n}} \int_{0}^{\frac{\rho+\varepsilon}{b_{0}+c}} \frac{\left(1-y^{2}\right)^{\frac{n-1}{2}}}{\left(1-\frac{c y}{\rho+\varepsilon}\right)^{n+1}} d y,
\end{gathered}
$$

where $r_{D}$ is the radius of the base of the spherical cone in $(D \cup L)^{x_{t}}$. We put

$$
R=\frac{1}{n}\left(\frac{1}{b_{0}}+\frac{1}{a+h}\right) \frac{v_{n-1}}{r_{D}^{n-1}}-\frac{v_{n-1}}{(\rho+\varepsilon)^{n}} \int_{0}^{\frac{\rho+\varepsilon}{b_{0}+c}} \frac{\left(1-y^{2}\right)^{\frac{n-1}{2}}}{\left(1-\frac{c y}{\rho+\varepsilon}\right)^{n+1}} d y .
$$

Then, by Lemma 4 (ii), for $\varepsilon>0$

$$
\left|K^{x_{t}}\right| \geq \frac{(1-\varepsilon) v_{n}}{2^{\frac{n+1}{2}}(\rho+\varepsilon)^{n}\left(\frac{c}{\rho+\varepsilon}\right)^{\frac{n+1}{2}}\left(1-\frac{c}{\rho+\varepsilon}\right)^{\frac{n+1}{2}}}+R,
$$

provided that

$$
\frac{c}{\rho+\varepsilon}>\frac{1}{1+\frac{2 \varepsilon}{3 n}} .
$$

We choose $t$ so big that this holds. 
Hence

$$
\begin{aligned}
t=\frac{|K|\left|K^{x_{t}}\right|}{v_{n}^{2}} \geq\left(\frac{1}{2}\right)^{\frac{n+1}{2}}\left(\frac{(1-\varepsilon)|K|}{v_{n}}\right) \frac{(\rho+\varepsilon)^{-n}}{\left(\frac{c}{\rho+\varepsilon}\left(1-\frac{c}{\rho+\varepsilon}\right)\right)^{\frac{n+1}{2}}} \\
\quad \times\left\{1+\frac{2^{\frac{n+1}{2}} R(\rho+\varepsilon)^{n}\left(\frac{c}{\rho+\varepsilon}\right)^{\frac{n+1}{2}}\left(1-\frac{c}{\rho+\varepsilon}\right)^{\frac{n+1}{2}}}{(1-\varepsilon) v_{n}}\right\}
\end{aligned}
$$

and by (9), for some constant $d$,

$$
\begin{gathered}
\frac{t^{\frac{2}{n+1}}}{n}\langle x, N(x)\rangle\left(1-\left(\frac{\left\|x_{t}\right\|}{\|x\|}\right)^{n}\right) \\
\geq \frac{1}{2}\left(\frac{(1-\varepsilon)|K|}{v_{n}}\right)^{\frac{2}{n+1}}(\rho+\varepsilon)^{-\frac{n-1}{n+1}} \frac{\left(1+k\left(2 \frac{\left\|x-x_{t}\right\| \cos \theta}{\rho+\varepsilon}-\frac{\left\|x-x_{t}\right\|^{2}}{(\rho+\varepsilon)^{2}}\right)\right)^{-1}\left(1-d \cdot \frac{\left\|x-x_{t}\right\|}{\|x\|}\right)}{\left(1+\frac{\left\|x-x_{t}\right\|^{2}}{(\rho+\varepsilon)^{2}}-2 \frac{\left\|x-x_{t}\right\| \cos \theta}{\rho+\varepsilon}\right)^{\frac{1}{2}}\left(1-\frac{\left\|x-x_{t}\right\|}{2(\rho+\varepsilon) \cos \theta}\right)} \\
\times\left\{1+\frac{2^{\frac{n+1}{2}} R(\rho+\varepsilon)^{n}}{(1-\varepsilon) v_{n}}\left(1-\frac{c}{\rho+\varepsilon}\right)^{\frac{n+1}{2}}\left(\frac{c}{\rho+\varepsilon}\right)^{\frac{n+1}{2}}\right\}^{\frac{2}{n+1}},
\end{gathered}
$$

as

$$
1-\frac{c}{\rho+\varepsilon} \leq \frac{\left\|x-x_{t}\right\| \cos \theta}{\rho+\varepsilon}\left(1-\frac{\left\|x-x_{t}\right\|}{2(\rho+\varepsilon) \cos \theta}\right)\left(1+k\left(2 \frac{\left\|x x_{t}\right\| \cos \theta}{\rho+\varepsilon}-\frac{\left\|x-x_{t}\right\|^{2}}{(\rho+\varepsilon)^{2}}\right)\right),
$$

for some constant $k$, if $t$ is big enough. $R$ remains bounded for $t \rightarrow \infty$.

Note also that $\cos \theta$ remains bounded from below by (8).

Thus we have a lower bound for the expression in question.

To get an upper bound we proceed in a similar way. For $\lambda \in \mathbf{R}$, now let $G_{\lambda}=\left\{x:\left\langle x, z_{\rho-\varepsilon}-x_{t}\right\rangle=\lambda\right\}$ be a hyperplane orthogonal to the line segment $\left[x_{t}, z_{\rho-\varepsilon}\right]$. Let $\lambda_{0}=\max \left\{\lambda: G_{\lambda}^{+} \cap B_{\rho \varepsilon} \subseteq H_{s_{0}}^{+} \cap B_{\rho-\varepsilon}\right\}$. Let $P$ be the point where the half-line starting at $x_{t}$ through $z_{\rho-\varepsilon}$ intersects $\partial K$.

Let $C$ be the spherical cone $C=\operatorname{co}\left[P, B_{\rho-\varepsilon} \cap G_{\lambda_{0}}\right]$. Let $h$ be the height of this cone (see Figure 3).

Let $L=\left\{x \in B_{\rho-\varepsilon}:\left\langle x, z_{\rho-\varepsilon}-x_{t}\right\rangle \leq \lambda_{0}\right\}$. Then $K \supseteq C \cup L$, and hence

$$
K^{x_{t}} \subseteq(C \cup L)^{x_{t}},
$$

and to estimate $\left|K^{x_{t}}\right|$ we have to compute $(C \cup L)^{x_{t}}$.

To do so we prove the more general

Claim 2. Let $M$ be the union of a spherical cone $C$ with height $h$ and a cap $L$ of a Euclidean ball with radius $r$ such that the base of $C$ coincides with the base of $L$. For a point $x$ in $L$ and on the axis of symmetry of $M$ let $\alpha=\operatorname{distance}(x, C)$, $\beta=\operatorname{distance}(x, \partial L)$ (see Figure 4). Then, with $\beta_{0}=\frac{r \beta+(\alpha+\beta)(r-\beta)}{r(\alpha+\beta)}$ and $x$ chosen such that $r>\alpha+\beta$,

$$
\begin{gathered}
\left|M^{x}\right|=v_{n-1}\left(\frac{1}{r^{n}} \int_{\frac{r}{(r-\beta)+\beta_{0}}}^{1} \frac{\left(1-y^{2}\right)^{\frac{n-1}{2}}}{\left(1-\frac{(r-\beta) y}{r}\right)^{n+1}} d y\right. \\
\left.+\frac{1}{n \alpha\left(2 r(\alpha+\beta)-(\alpha+\beta)^{2}\right)^{\frac{n 1}{2}}}\left(\frac{\left(\alpha+\beta_{0}\right)^{n}}{\beta_{0}^{n}}-\frac{h^{n}}{(\alpha+h)^{n}}\right)\right) .
\end{gathered}
$$




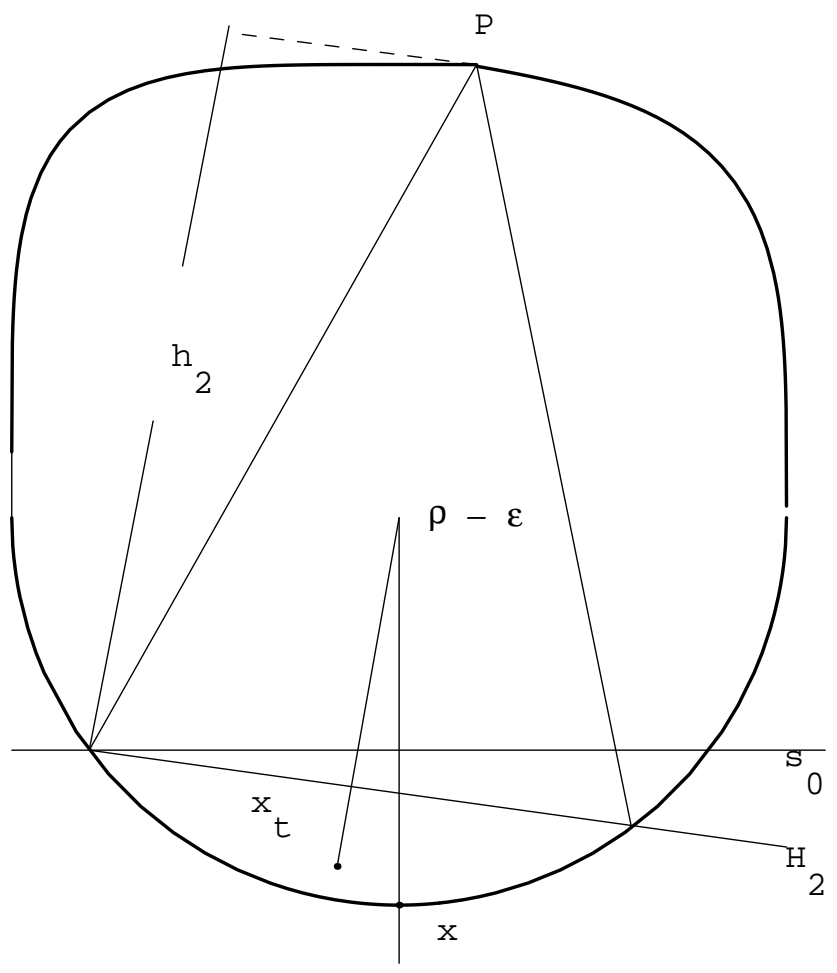

Figure 3 . The estimate from above

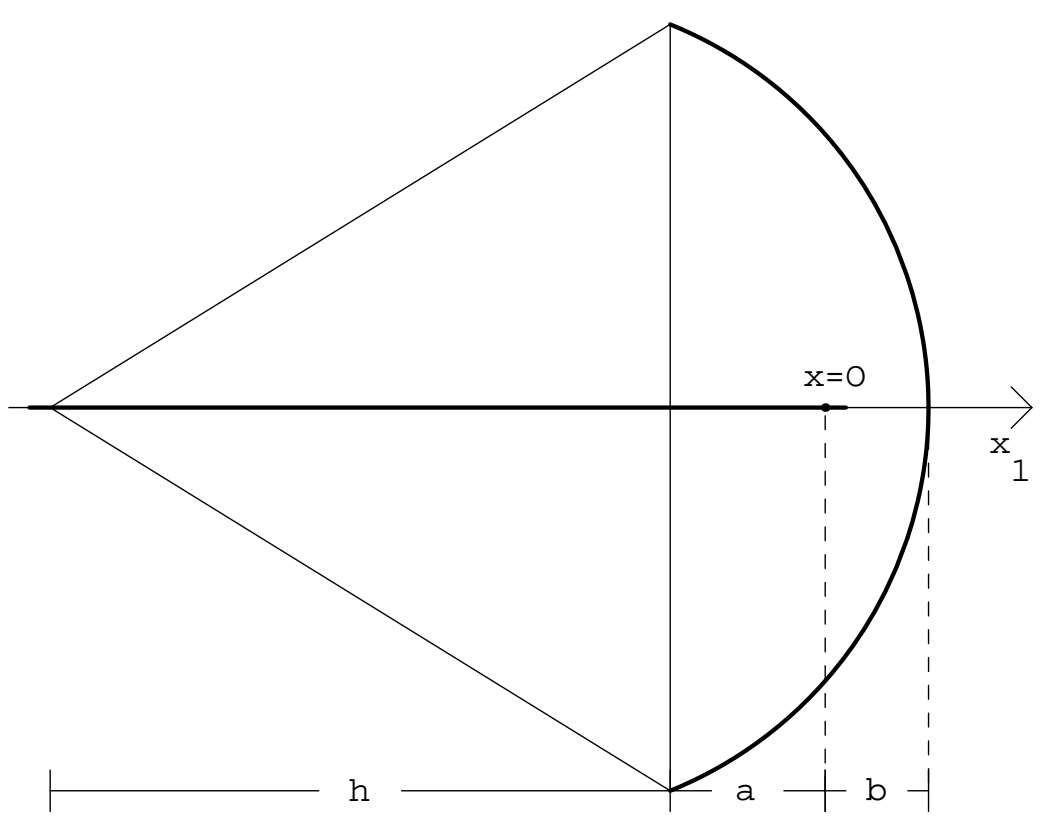

Figure 4. Claim 2 
Proof of Claim 2. We introduce a coordinate system such that $x=0$ and the $x_{1^{-}}$ axis coincides with the axis of symmetry of $M$ (see Figure 4).

Notice now that $M^{0}$ is such that each $(n-1)$-dimensional section orthogonal to the $x_{1}$-axis is an $(n-1)$-dimensional Euclidean ball with radius $l\left(x_{1}\right)$, where

$$
\begin{gathered}
l\left(x_{1}\right)=\frac{\alpha x_{1}+1}{\left(2 r(\alpha+\beta)-(\alpha+\beta)^{2}\right)^{\frac{1}{2}}}, \quad \text { if } \quad-\frac{1}{\alpha+h} \leq x_{1} \leq \frac{1}{\beta_{0}}, \\
l\left(x_{1}\right)=\frac{1}{r}\left(\left(1+x_{1}(r-\beta)\right)^{2}-r^{2} x_{1}^{2}\right)^{\frac{1}{2}}, \quad \text { if } \quad \frac{1}{\beta_{0}} \leq x_{1} \leq \frac{1}{\beta} .
\end{gathered}
$$

From this Claim 2 follows.

Now we apply Claim 2 to our situation. There

$$
r=\rho-\varepsilon, \beta=\rho-\varepsilon-\gamma,
$$

where

$$
\begin{gathered}
\gamma^{2}=\left\|x-x_{t}\right\|^{2}+(\rho-\varepsilon)^{2}-2(\rho-\varepsilon)\left\|x-x_{t}\right\| \cos \theta \\
\alpha=\gamma-\frac{\rho-\varepsilon-s_{0}}{\gamma}\left(\gamma^{2}-\left\|x-x_{t}\right\|^{2} \sin ^{2} \theta\right)^{\frac{1}{2}}-\frac{\left(2(\rho-\varepsilon) s_{0}-s_{0}^{2}\right)^{\frac{1}{2}}\left\|x-x_{t}\right\| \sin \theta}{\gamma}
\end{gathered}
$$

and

$$
\beta_{0}=\frac{(\rho-\varepsilon)^{2}}{\gamma-\alpha}-\gamma
$$

Then we get, similarily as before,

$$
\begin{gathered}
\frac{t^{\frac{2}{n+1}}}{n}\langle x, N(x)\rangle\left(1-\left(\frac{\left\|x_{t}\right\|}{\|x\|}\right)^{n}\right) \\
\leq \frac{1}{2}\left(\frac{|K|}{v_{n}}\right)^{\frac{2}{n+1}}(\rho-\varepsilon)^{-\frac{n-1}{n+1}} \frac{1}{\left(1+\frac{\left\|x-x_{t}\right\|^{2}}{(\rho-\varepsilon)^{2}}-2 \frac{\left\|x-x_{t}\right\| \cos \theta}{\rho-\varepsilon}\right)^{\frac{1}{2}}\left(1-\frac{\left\|x-x_{t}\right\|}{2(\rho-\varepsilon) \cos \theta}\right)} \\
\times\left\{1+\frac{2^{\frac{n+1}{2}} R(\rho-\varepsilon)^{n}}{v_{n}}\left(1-\frac{\gamma}{\rho-\varepsilon}\right)^{\frac{n+1}{2}}\left(\frac{\gamma}{\rho-\varepsilon}\right)^{\frac{n+1}{2}}\right\}^{\frac{2}{n+1}},
\end{gathered}
$$

with a suitably defined $R$.

This finishes the proof of Lemma 13 in the case where the indicatrix is an ellipsoid.

(ii) Case where the Dupin indicatix is an elliptic cylinder. Recall that then we have to show that

$$
\lim _{t \rightarrow \infty} \frac{1}{n}\langle x, N(x)\rangle t^{\frac{2}{n+1}}\left(1-\left(\frac{\left\|x_{t}\right\|}{\|x\|}\right)^{n}\right)=0 .
$$

We can again assume (see $[\mathrm{S}-\mathrm{W}]$ ) that the indicatrix is a spherical cylinder, i.e. the product of a $k$-dimensional plane and an $(n-k-1)$-dimensional Euclidean sphere of radius $\rho$. Moreover we can assume that $\rho$ is arbitrarily large (see also $[\mathrm{S}-\mathrm{W}]$ ).

By Lemma 9 of $[\mathrm{S}-\mathrm{W}]$ we then have, for sufficiently small $s$ and some $\varepsilon>0$,

$$
B_{\rho-\varepsilon} \cap H_{s}^{+} \subseteq K \cap H_{s}^{+} .
$$

Using similar methods, this implies Lemma 13. 
Proposition 14. Let $K$ be a convex body such that $\partial K$ is $C^{3}$ and has strictly positive Gaussian curvature everywhere. Then there is $\delta_{0}>0$ such that for all $\delta<\delta_{0}$

$$
S\left(K, \frac{v_{n-1}}{2(n+1) v_{n} \delta}\right) \subseteq K_{\delta|K|} .
$$

Proof. As in the proof of Lemma 12, we can choose $1>\alpha>0$ such that

$$
B(0, \alpha) \subseteq K \subseteq B\left(0, \frac{1}{\alpha}\right) .
$$

Therefore we have, for all $x \in \partial K$,

$$
1 \geq\left\langle\frac{x}{\|x\|}, N(x)\right\rangle \geq \alpha^{2} .
$$

Let $R_{0}=\min _{x \in \partial K, 1 \leq i \leq n-1} R_{i}(x)$, where $R_{i}(x)$ is the $i$-th principal radius of curvature at $x \in \partial K$. We know that $R_{0}>0$ (see [L2]).

Let $1>\varepsilon>0$ be given such that $\varepsilon<\min \left\{\frac{R_{0}}{2}, 6 n \alpha^{4}\right\}$ and

$$
(1-\varepsilon)\left(1-\frac{\varepsilon}{R_{0}}\right)^{n-1}\left(1-\frac{\varepsilon}{6 n \alpha^{4}}\right)^{n}>\frac{1}{2} .
$$

By assumption the Dupin indicatrix exists for all $x \in \partial K$ and is an ellipsoid. For $x \in \partial K$ given, we can assume that, after an affine transformation, the indicatrix at $x$ is a Euclidean sphere. Let $\sqrt{\rho(x)}$ be the radius of this Euclidean sphere. Note that, for all $x \in \partial K$,

$$
\rho(x) \geq R_{0} .
$$

Then, with the notation used in the proof of Lemma 13, there exists $s(x)>0$ such that

$$
B_{\rho(x)-\varepsilon} \cap H_{s(x)}^{+} \subseteq K \cap H_{s(x)}^{+} \subseteq B_{\rho(x)+\varepsilon} \cap H_{s(x)}^{+} .
$$

Let $s_{1}=\min _{x \in \partial K} s(x)$. We know that $s_{1}>0$, as $\partial K$ is $C^{3}$ and compact. Let

$$
s_{0}=\min \left\{s_{1},\left(R_{0}-\varepsilon\right)\left(1-\frac{1}{1+\frac{2 \varepsilon}{3 n}}\right)\right\} .
$$

Let $\delta_{0}>0$ be so small that for all $x \in \partial K$ two conditions are satisfied: first

$$
\left\|x-x_{\delta_{0}}\right\|\left\langle\frac{x}{\|x\|}, N(x)\right\rangle \leq \frac{s_{0}}{2},
$$

where $x_{\delta_{0}}=[0, x] \cap \partial K_{\delta_{0}|K|}$, and, second,

$$
H_{\delta_{0}}^{+} \cap B_{\rho(x)-\varepsilon} \subseteq H_{s_{0}}^{+} \cap B_{\rho(x)-\varepsilon},
$$

where $H_{\delta_{0}}$ is the hyperplane through $x_{\delta_{0}}$ that cuts off exactly $\delta_{0}|K|$ from $K$.

Suppose now that the above proposition is not true. Then there is $\delta<\delta_{0}$ and $x_{s} \in \partial S\left(K, \frac{v_{n-1}}{2(n+1) v_{n} \delta}\right)$ such that $x_{s} \notin K_{\delta|K|}$. Let $x \in \partial K$ be such that $x_{s} \in[0, x]$. We also can assume that the Dupin indicatrix at $x$ is a Euclidean ball with radius $\sqrt{\rho(x)}$. We choose $x_{\delta} \in \partial K_{\delta|K|}$ such that $x_{\delta} \in[0, x]$. Then

$$
\left\|x-x_{\delta}\right\|\left\langle\frac{x}{\|x\|}, N(x)\right\rangle<\left\|x-x_{s}\right\|\left\langle\frac{x}{\|x\|}, N(x)\right\rangle .
$$

By construction

$$
\begin{gathered}
\delta|K|=\left|K \cap H_{\delta}^{+}\right| \geq\left|B_{\rho(x)-\varepsilon} \cap H_{\delta}^{+}\right| \\
\geq \min _{H \in \mathcal{H}}\left|B_{\rho(x)-\varepsilon} \cap H^{+}\right|=\left|B_{\rho(x)-\varepsilon} \cap H_{0}^{+}\right|,
\end{gathered}
$$


where

$$
\mathcal{H}=\left\{H: H \text { is a hyperplane through } x_{\delta}, x \in H^{+}\right\} .
$$

For the height $h$ of this cap $\left|B_{\rho(x)-\varepsilon} \cap H_{0}^{+}\right|$of $B_{\rho(x)-\varepsilon}$ of minimal volume we have

$$
h \geq\left\|x-x_{\delta}\right\|\left\langle\frac{x}{\|x\|}, N(x)\right\rangle\left(1-\frac{\left\|x-x_{\delta}\right\|}{2\left\langle\frac{x}{\|x\|}, N(x)\right\rangle(\rho(x)-\varepsilon)}\right) .
$$

Using (10), (11) and (12), we get

$$
h \geq\left\|x-x_{\delta}\right\|\left\langle\frac{x}{\|x\|}, N(x)\right\rangle\left(1-\frac{\varepsilon}{6 n \alpha^{4}}\right) .
$$

The volume of a cap of a Euclidean ball with radius $r$ and height $h$ can be estimated from below by

$$
\geq \frac{v_{n-1} 2^{\frac{n+1}{2}} r^{\frac{n-1}{2}} h^{\frac{n+1}{2}}}{n+1}\left(1-\frac{h}{2 r}\right)^{\frac{n+1}{2}} .
$$

Therefore

$$
\delta|K| \geq \frac{v_{n-1}}{n+1} 2^{\frac{n+1}{2}} \rho(x)^{\frac{n-1}{2}}\left(\left\|x-x_{\delta}\right\|\left\langle\frac{x}{\|x\|}, N(x)\right\rangle\right)^{\frac{n+1}{2}}\left(1-\frac{\varepsilon}{R_{0}}\right)^{\frac{n-1}{2}}\left(1-\frac{\varepsilon}{6 n \alpha^{4}}\right)^{n},
$$

where we have used again (10), (11), (12) and the fact that $\left\langle\frac{x}{\|x\|}, N(x)\right\rangle \geq \alpha^{2}$. Thus

$$
\left(\left\|x-x_{s}\right\|\left\langle\frac{x}{\|x\|}, N(x)\right\rangle\right)^{\frac{n+1}{2}}<\frac{(n+1) \delta|K| \rho(x)^{-\frac{n-1}{2}}}{v_{n-1} 2^{\frac{n+1}{2}}\left(1-\frac{\varepsilon}{6 n \alpha^{4}}\right)^{n}\left(1-\frac{\varepsilon}{R_{0}}\right)^{\frac{n-1}{2}}} .
$$

Since, in the notation of Lemma 13 and using (10), (11) and (12),

$$
\frac{c}{\rho(x)+\varepsilon} \geq 1-\frac{\left\|x-x_{s}\right\|\left\langle\frac{x}{\|x\|}, N(x)\right\rangle}{\rho(x)+\varepsilon}>\frac{1}{1+\frac{2 \varepsilon}{3 n}},
$$

the estimate from below from Lemma 13 for $|K|\left|K^{x}\right| / v_{n}^{2}$ holds for $x=x_{s}$, and we get (see p. 17)

$$
\frac{|K|\left|K^{x_{s}}\right|}{v_{n}^{2}}>\frac{(1-\varepsilon)|K| \rho(x)^{-n}\left(1+\frac{\varepsilon}{\rho(x)}\right)^{-n}}{2^{\frac{n+1}{2}} v_{n}\left(\frac{c}{\rho(x)+\varepsilon}\right)^{\frac{n+1}{2}}\left(1-\frac{c}{\rho(x)+\varepsilon}\right)^{\frac{n+1}{2}}} .
$$

Now notice that

$$
\frac{c}{\rho(x)+\varepsilon} \leq 1
$$

and

$$
1-\frac{c}{\rho(x)+\varepsilon} \leq \frac{\left\|x-x_{s}\right\|\left\langle\frac{x}{\|x\|}, N(x)\right\rangle}{\rho(x)\left(1+\frac{\varepsilon}{\rho(x)}\right.} .
$$

Therefore (13) implies that

$$
\frac{|K|\left|K^{x_{s}}\right|}{v_{n}^{2}}>(1-\varepsilon)\left(1-\frac{\varepsilon}{R_{0}}\right)^{n-1}\left(1-\frac{\varepsilon}{6 n \alpha^{4}}\right)^{n} \frac{v_{n-1}}{(n+1) \delta v_{n}} .
$$

This is a contradiction. 


\section{REFERENCES}

[B] K. Ball: Logarithmically concave functions and sections of convex sets in $\mathbf{R}^{n}$, Studia Math. vol. 88 (1988),69-84. MR 89e:52002

[D-H] G. Dolzmann, D. Hug: Equality of two representations of extended affine surface area, Archiv der Mathematik 65 no.4 (1995),352-356. MR 97c:52019

[F] M. Fradelizi: Hyperplane sections of convex bodies in isotropic position, to appear in Contributions to Algebra and Geometry.

[G] P. Gruber: Aspects of approximation of convex bodies, Handbook of Convex Geometry, vol.A(1993), 321-345, North Holland. MR 95b:52003

[He] D. Hensley: Slicing convex bodies - bounds for slice area in terms of the body's covariance, Proc. Amer. Math. Soc. 79(4) (1980), 619-625. MR 81j:52008

[L1] K. Leichtweiss: Zur Affinoberfläche konvexer Körper, Manuscripta Mathematica 56 (1986), 429-464. MR 87k:52011

[L2] K. Leichtweiss: Über eine Formel Blaschkes zur Affinoberfläche, Studia Scient. Math. Hung. 21 (1986), 453-474. MR 89b:53016

[Lu] E. Lutwak: Extended affine surface area, Adv. in Math. 85 (1991), 39-68. MR 92d:52012

[Lu-O] E. Lutwak, V.Oliker: On the regularity of solutions to a generalization of the Minkowski problem, J. Differential Geometry, vol. 41 (1995), 227-246. MR 95m:52007

[Me-P] M. Meyer, A. Pajor: On the Blaschke Santaló inequality, Arch. Math. 55(1990), 82-93. MR 92b:52013

[Mi-P] V. D. Milman, A. Pajor: Isotropic position and inertia ellipsoids and zonoids of the unit ball of a normed $n$-dimensional space, GAFA (eds. J. Lindenstrauss, V.D. Milman), Lecture Notes in Math.1376, Springer, Berlin (1989), 64-104. MR 90g:52003

[Sch] R. Schneider: Convex bodies: The Brunn-Minkowski theory, Encyclopedia of Math. and its Applic.44, Cambridge University Press (1993). MR 94d:52007

[S1] C. Schütt: On the affine surface area, Proc. Amer. Math. Soc. 118 (1993), 1213-1218. MR 93j:52009

[S2] C. Schütt: Floating body, illumination body and polytopal approximation, C. R. Acad. Sci. Paris Sér. I Math. 324 (1997), 201-203. MR 98b:52005

[S-W] C. Schütt, E. Werner: The convex floating body, Math. Scand. 66 (1990), 275-290. MR 91i: 52005

[W] E. Werner: Illumination bodies and affine surface area, Studia Math. 110 (1994), 257269. MR 95g:52010

Université de Marne-La-Valee, Equipe d'Analyse et de Mathématiques Appliquees, Cité Descartes-5, Bd Descartes-Champs-sur-Marne, 77454 Marne-la-Vallée cedex 2, FRANCE

E-mail address: meyer@math.univ-mlv.fr

Department of Mathematics, Case Western Reserve University, Cleveland, Ohio 44106

E-mail address: emw2@po.cwru.edu

Current address: Université de Lille 1, UFR de Mathématiques, 59655 Villeneuve d'Ascq, France 